This document is published in:

Economics Letters, (2006), 90 (3), 348-355.

DOI: http://dx.doi.org/10.1016/j.econlet.2005.08.028

(C) 2005 Elsevier B.V. 


\title{
Temptation, self-control, and competitive nonlinear pricing
}

\author{
Susanna Esteban $^{\mathrm{a}, *}$, Eiichi Miyagawa ${ }^{\mathrm{b}}$ \\ ${ }^{a}$ Department of Economics, Pennsylvania State University, University Park, PA 16802, United States \\ ${ }^{\mathrm{b}}$ Department of Economics, Columbia University, New York, NY 10027, United States
}

\begin{abstract}
Standard pricing theories consider consumers without temptation. With temptation and costly self-control, consumers dislike choice sets with tempting alternatives. We study firms' strategy against such consumers, using Gul-Pesendorfer preferences and a game where firms compete by offering menus.
\end{abstract}

Keywords: Temptation; Self-control; Nonlinear pricing; Pooling; Participation fees

JEL classification: D43; L13; L15

\section{Introduction}

The standard theories of optimal pricing by firms are based on the assumption that consumers are free from temptation. On the other hand, temptation is undoubtedly an important feature of consumers' behavior, and there is a recent theoretical advance that allows us to study consumption decisions with temptation. In a recent paper, Gul and Pesendorfer (2001) axiomatically derived a class of preferences under which consumers are subject to temptation and incur psychological costs if they exercise selfcontrol. We use Gul and Pesendorfer's formulation to study firms' optimal pricing when consumers are subject to temptation. Unlike consumers with standard preferences, consumers with Gul-Pesendorfer preferences may dislike a larger choice set if it contains tempting and undesirable choices, since

\footnotetext{
* Corresponding author. Tel.: +1 814865 0010; fax: +1 8148634775.

E-mail address: sesteban@psu.edu (S. Esteban).
} 
consumers may succumb to temptation and even if they resist it, they incur psychological costs in the process.

In the Gul-Pesendorfer formulation, each consumer has two utility functions, one representing preferences that she would like to commit to, and the other one representing her temptation. The consumer's actual choice is determined as a compromise between the two utility functions, and the compromising process captures the exercise of self-control. The psychological cost of self-control is determined endogenously as the forgone utility of temptation: the difference between the maximum temptation utility and the temptation utility of the actual choice. Consumers correctly foresee the outcome of their self-control. If a consumer expects that the self-control process will be too painful or little self-control will be exercised, she may choose to stay away from the purchasing opportunity.

If consumers behave in this way, what are the implications for the firms' optimal strategies? To answer this question, we study the standard framework of nonlinear pricing due to Mussa and Rosen (1978) and Maskin and Riley (1984) and consider the case where a set of firms compete. ${ }^{1}$ Since consumers have a preference for small, less tempting menus, firms may want to offer multiple menus and separate consumers into them via self-selection. Thus we study a game in which firms can offer a set of menus.

We characterize the Nash equilibria of the game. An interesting feature of the equilibrium is that even though the market is extremely competitive, the equilibrium outcome is generally not efficient in the following sense: once a consumer chooses her most preferred menu, she and the firm can find a Pareto improving alternative outside the menu. The Pareto improving alternative (good price pair) is not offered in equilibrium since it is not desirable for the consumer's commitment utility and if offered, the consumer would stay away from the menu. We also show that, in contrast with the standard model, the equilibrium involves pooling of consumers. Consumers with non-trivially different preferences choose the same menu and the same item from the menu.

The last section of the paper lists a few more properties of the equilibria.

Our companion papers (Esteban et al., 2003; Esteban and Miyagawa, 2004) study the same framework and consider the case of monopoly. The former considers the case where the monopolist is restricted to offering one menu, while the latter considers the case where the firm can offer any number of menus.

\section{Menus}

There is a set of goods indexed by their quality level (or quantity) $q \in \mathbb{R}_{+}$. The good $q=0$ represents "nothing." Consumers are interested in buying at most one unit of one good. An offer is a pair $(q, t) \in \mathbb{R}_{+}^{2}$, which represents the option of buying one unit of good $q$ for a price of $t$. A menu is a set of offers $M \subseteq \mathbb{R}_{+}^{2}$ such that $(0,0) \in M$. The restriction $(0,0) \in M$ means that a consumer who buys nothing pays nothing. We limit ourselves to menus that are compact sets.

\section{Firms}

There are $n \geq 2$ firms and the set of firms is denoted $N=\{1,2, \ldots, n\}$. A firm can offer any number of menus. As in standard theory, it is costless to create a menu. For example, a menu may be a cellphone

\footnotetext{
${ }^{1}$ Economides (1985) and Mandy (1992) study similar games with standard preferences of consumers.
} 
plan, where $q$ is the number of minutes of calls, or a weight-loss program plan, where $q$ is the amount of weight loss. For these examples, creating a menu does not involve any significant physical activities.

Another possible interpretation is that each menu represents an individual retail store. A firm can open multiple stores, possibly under different brand names, that target different ranges of consumers. The assumption that menus can be created costlessly captures an extreme form of competitive markets, and it is useful as it allows us to focus on the multiplicity of menus that arises from consumers' preferences.

Let $C: \mathbb{R}_{+} \rightarrow \mathbb{R}_{+}$be the cost function, where $C(q)$ denotes the per-consumer cost of producing good $q$. The cost function $C$ is identical for all firms, strictly increasing, convex, and satisfies $C(0)=0$. For each offer $x=(q, t)$, let $\Pi(x) \equiv t-C(q)$ denote the per-consumer profit generated by the offer.

\section{Consumers}

Let $\Theta$ be a finite set of consumer types. For each $\theta \in \Theta$, let $f(\theta)>0$ denote the fraction of consumers of type $\theta$. The firms know $f$ but not the type of each consumer. Consumers know the set of menus offered by each firm. Following Gul and Pesendorfer (2001), we assume that consumers have the following utility function defined over menus:

$$
W_{\theta}(M) \equiv \max _{(q, t) \in M}\left[U_{\theta}(q, t)+V_{\theta}(q, t)\right]-\max _{(q, t) \in M} V_{\theta}(q, t),
$$

where $U_{\theta}$ and $V_{\theta}$ are functions from $\mathbb{R}_{+}^{2}$ into $\mathbb{R}$. The function $U_{\theta}$ represents the preferences that the consumer would like to commit to, while $V_{\theta}$ represents her temptation. The offer that the consumer actually chooses is one that maximizes $U_{\theta}+V_{\theta}$, which represents a compromise between her commitment preferences and her temptation preferences. The relative power of each preference ranking in determining the consumer's choice depends on the relative scale of $U_{\theta}$ and $V_{\theta}$. For instance, increasing the relative scale of $U_{\theta}$ (e.g., by multiplying it by a constant number $\alpha>1$ ) increases the consumer's "willpower." Let $\mathrm{BO}_{\theta}(M)$ denote the set of offers that maximize $U_{\theta}+V_{\theta}$ :

$$
\mathrm{BO}_{\theta}(M)=\underset{x \in M}{\operatorname{Argmax}}\left[U_{\theta}(x)+V_{\theta}(x)\right]
$$

The solutions to the second maximization in Eq. (1) represent the most tempting offers. Thus, if the consumer chooses $\hat{x} \in \mathrm{BO}_{\theta}(M)$, the difference

$$
\max _{x \in M} V_{\theta}(x)-V_{\theta}(\hat{x})
$$

is the forgone utility of $V_{\theta}$. In terms of temptation preferences, the consumer wants to maximize $V_{\theta}$ but ends up with $\hat{x}$ after self-control. Therefore, the difference in Eq. (3) can be interpreted as the consumer's disutility from self-control and is called the self-control cost. With this terminology, the consumer's overall utility equals $U_{\theta}(\hat{x})$ minus the self-control cost. Because of the self-control cost, consumers have an incentive to avoid menus that contain tempting offers.

A critical feature of the consumers' behavior is that consumers choose an offer in two stages. A consumer first chooses a menu $M$ and then an offer $x \in M$. When she chooses a menu, she maximizes $W_{\theta}(M)$. When she chooses an offer from $M$, she maximizes $U_{\theta}(x)+V_{\theta}(x)$. The first stage is called the exante stage, and the second stage is called the ex-post stage. Similarly, $W_{\theta}(M)$ is called the ex-ante utility, and $U_{\theta}(x)+V_{\theta}(x)$ is called the ex-post utility. 
We assume that $U_{\theta}$ and $V_{\theta}$ are continuous, strictly increasing in $q$, strictly decreasing in $t$, strictly quasi-concave, ${ }^{2}$ and satisfy $U_{\theta}(0,0)=V_{\theta}(0,0)=0$. We also assume that for all $\theta$,

$$
\max _{q \geq 0} U_{\theta}(q, C(q)) \text { and } \max _{q \geq 0} V_{\theta}(q, C(q))
$$

have strictly positive solutions.

\section{Game}

We consider a normal-form game in which each firm can choose any number of menus. Formally, each firm $i$ chooses a finite set of indexes $S_{i} \subseteq\{1,2, \ldots$,$\} and a list of menus \left(M_{i}^{s}\right)_{s \in S_{i}}$.

Given the firms' strategies, the set of all menus offered by the firms is $\mathcal{M}=\left\{M_{i}^{s}: i \in N\right.$ and $\left.s \in S_{i}\right\}$. Let $\mathrm{BM}_{\theta}(\mathcal{M})$ be the set of menus $M \in \mathcal{M}$ such that

$$
\begin{aligned}
& W_{\theta}(M) \geq 0, \\
& W_{\theta}(M) \geq W_{\theta}\left(M^{\prime}\right) \text { for all } M^{\prime} \in \mathcal{M},
\end{aligned}
$$

where Eq. (5) is the ex-ante individual rationality condition and Eq. (6) is the ex-ante incentive compatibility condition. For consumers $\theta$, all menus in $\mathrm{BM}_{\theta}(\mathcal{M})$ are equally good and hence we assume that the consumers choose each of these menus with equal probability. Upon choosing a menu, the most preferred offers in the menu for the consumer may not be unique. In this case, the consumer chooses one that is most profitable for the firm. If multiple such offers exist, which one is chosen is immaterial. Formally, let $\mathrm{BO}_{\theta}^{*}(M)$ be an offer $x^{*} \in \mathrm{BO}_{\theta}(M)$ such that $\Pi\left(x^{*}\right) \geq \Pi(x)$ for all $x \in \mathrm{BO}_{\theta}(M)$.

We can now define firms' payoffs. Given a strategy profile $\left(S_{i},\left(M_{i}^{S}\right)_{s \in S_{i}}\right)_{i \in N}$ and the associated set of menus $\mathcal{M}$, firm $i$ 's payoff is

$$
\pi_{i}(\mathcal{M})=\sum_{s \in S_{i}} \sum_{\substack{\theta \in \Theta s . t . \\ M_{i}^{s} \in B M_{\theta}(\mathcal{M})}}[t(\theta, s)-C(q(\theta, s))] \frac{f(\theta)}{\left|\mathrm{BM}_{\theta}(\mathcal{M})\right|}
$$

where, for all $(\theta, s) \in \Theta \times S_{i}$ such that $M_{i}^{s} \in \mathrm{BM}_{\theta}(\mathcal{M}), \quad(q(\theta, s), \quad t(\theta, s))=\mathrm{BO}_{\theta}^{*}\left(M_{i}^{s}\right)$. In words, $M_{i}^{s} \in \mathrm{BM}_{\theta}(\mathcal{M})$ is a most preferred menu for consumers $\theta$, so some of these consumers choose the menu. Since there exist $\left|\mathrm{BM}_{\theta}(\mathcal{M})\right|$ menus that are equally optimal for the consumers, only a fraction $1 /\left|\mathrm{BM}_{\theta}(\mathcal{M})\right|$ of them choose $M_{i}^{s}$. Those who choose $M_{i}^{s}$ choose the offer $(q(\theta, s), t(\theta, s))=\mathrm{BO}_{\theta}^{*}\left(M_{i}^{s}\right)$ and generate profits $t(\theta, s)-C(q(\theta, s))$ per consumer.

\section{Equilibria}

We characterize the Nash equilibria of the game defined above. Let $\left(S_{i},\left(\left(M_{i}^{S}\right)_{S \in S_{i}}\right)_{i \in N}\right.$ be a Nash equilibrium and $\mathcal{M}$ be the associated set of menus. Let $\pi_{i}$ denote firm $i$ 's profit at the equilibrium. Since not offering any menu is an option, $\pi_{i} \geq 0$.

\footnotetext{
${ }^{2}$ As usual, if $C$ is strictly convex, we can allow for weak quasi-concavity of $U_{\theta}$ and $V_{\theta}$.
} 
Lemma 1. All firms earn zero profits: $\pi_{i}=0$ for all $i$.

Proof. Let $j$ be a firm with the lowest profit and suppose the firm offers a replica of all the existing menus of all the firms. Since half of the consumers switch to the newly offered menus, firm $j$ earns $\left[\pi_{j}+\sum_{i \in N} \pi_{i}\right] / 2$. Since this should not exceed $\pi_{j}$, we have $\sum_{k \neq j} \pi_{k}=0$.

Lemma 2. Any offer that a consumer actually chooses generates zero profit: For all $i \in N$, all $s \in S_{i}$, and all $\theta \in \Theta$, if $M_{i}^{s} \in B M_{\theta}(\mathcal{M})$, then $\Pi\left(B O_{\theta}^{*}\left(M_{i}^{s}\right)\right)=0$.

Proof. If $\Pi\left(\mathrm{BO}_{\theta}^{*}\left(M_{i}^{s}\right)\right)>0$, firm $i$ gains by replacing all its menus with one that offers $\mathrm{BO}_{\theta}^{*}\left(M_{i}^{s}\right)$ only (i.e., $\left.\left\{\mathrm{BO}_{\theta}^{*}\left(M_{i}^{s}\right),(0,0)\right\}\right)$. Indeed, since the new menu is at least as good as $M_{i}^{s}$ for consumers $\theta$, the menu is chosen by some consumers, and hence the firm earns positive profits, which is the desired contradiction.

To characterize the optimal menu, let $x_{\theta}^{*}$ for $\theta$ each be an offer that maximizes

$$
H_{\theta}(x) \equiv U_{\theta}(x)+V_{\theta}(x)-\max \left\{0, V_{\theta}(x)\right\}
$$

subject to $\Pi(x) \leq 0$. Since $U_{\theta}$ and $V_{\theta}$ are strictly quasi-concave, so is Eq. (8). Thus $x_{\theta}^{*}$ is unique for each $\theta$, if it exists. By Assumption Eq. (4), $x_{\theta}^{*}$ exists and $H_{\theta}\left(x_{\theta}^{*}\right)>0$.

The offer $x_{\theta}^{*}$ can be found as follows. Let $x_{\theta}^{U}$ be the solution to

$$
\max _{x} U_{\theta}(x) \quad \text { s.t. } \Pi(x)=0 .
$$

If $V_{\theta}\left(x_{\theta}^{U}\right) \geq 0$, then $x_{\theta}^{*}=x_{\theta}^{U}$. Otherwise, let $x_{\theta}^{U+V}$ be the solution to

$$
\max _{x} U_{\theta}(x)+V_{\theta}(x) \quad \text { s.t. } \Pi(x)=0 .
$$

If $V_{\theta}\left(x_{\theta}^{U+V}\right) \leq 0$, then $x_{\theta}^{*}=x_{\theta}^{U+V}$. Otherwise, $x_{\theta}^{*}$ is the unique non-zero offer defined by

$$
V_{\theta}\left(x_{\theta}^{*}\right)=0 \text { and } \Pi\left(x_{\theta}^{*}\right)=0 .
$$

Here is an alternative procedure to identify $x_{\theta}^{*}$. Let $q_{\theta}^{U}, q_{\theta}^{U+V}$, and $q_{\theta}^{V}$ be the solution to $\max _{q} F_{\theta}(q, C(q))$ for $F \in\{U, U+V, V\}$, respectively. If $q_{\theta}^{U}<q_{\theta}^{U+V}<q_{\theta}^{V}$, then $q_{\theta}^{*}=q_{\theta}^{U}$. If $q_{\theta}^{V}<q_{\theta}^{U+V}$, then $q_{\theta}^{*}=$ median $\left\{q_{\theta}^{U+V}, q_{\theta}^{U}, \hat{q}_{\theta}\right\}$, where $\hat{q}_{\theta} \neq 0$ is defined by $V_{\theta}\left(\hat{q}_{\theta}, C\left(\hat{q}_{\theta}\right)\right)=0$. Once $q_{\theta}^{*}$ is identified, $t_{\theta}^{*}$ is given by $t_{\theta}^{*}=C\left(q_{\theta}^{*}\right)$.

Proposition 1. For any Nash equilibrium and any type $\theta$, (a) the ex-ante utility of consumers of type $\theta$ is $H_{\theta}\left(x_{\theta}^{*}\right)$; (b) all consumers of type $\theta$ choose $x_{\theta}^{*}$; and (c) at least two firms offer menus $M$ such that $W_{\theta}(M)=H_{\theta}\left(x_{\theta}^{*}\right)$. Conversely, (d) a strategy profile is a Nash equilibrium if the set of menus is $\mathcal{M}=\left\{\left\{x_{\theta}^{*},(0,0)\right\}: \theta \in \Theta\right\}$ and, for each type $\theta$, there exist at least two firms that offer $\left\{x_{\theta}^{*},(0,0)\right\}$.

Proof. To prove the first statement, fix $\theta$. To prove $(a)$, let $M_{i}^{s} \in \mathrm{BM}_{\theta}(\mathcal{M})$ and $x_{\theta}=\mathrm{BO}_{\theta}^{*}\left(M_{i}^{s}\right)$.

Then

$$
W_{\theta}\left(M_{i}^{s}\right)=U_{\theta}\left(x_{\theta}\right)+V_{\theta}\left(x_{\theta}\right)-\max _{y \in M_{i}^{s}} V_{\theta}(y) \leq U_{\theta}\left(x_{\theta}\right)+V_{\theta}\left(x_{\theta}\right)-\max \left\{0, V_{\theta}\left(x_{\theta}\right)\right\} \leq H_{\theta}\left(x_{\theta}^{*}\right) .
$$

Suppose, by contradiction, that $W_{\theta}\left(M_{i}^{s}\right)<H_{\theta}\left(x_{\theta}^{*}\right)$. Then there exists an offer $\hat{x}$ such that $W_{\theta}\left(M_{i}^{S}\right)<H_{\theta}(\hat{x})$ and $\Pi(\hat{x})>0$. Thus any firm can earn positive profits by replacing all of its menus with $\{\hat{x}(0,0)\}$.

To prove $(b)$, note that by $(a)$, the inequalities in Eq. (12) hold with equality, which implies $x_{\theta}=x_{\theta}^{*}$. 
To prove $(c)$, suppose that only one firm, say $j$, offers a menu that gives type $\theta$ an ex-ante utility of $H_{\theta}\left(x_{\theta}^{*}\right)$. Thus, for all menus $M$ offered by the other firms, $W_{\theta}(M)<H_{\theta}\left(x_{\theta}^{*}\right)$. Then there exists an offer $\hat{x}$ such that $\Pi(\hat{x})>0$ and $H_{\theta}(\hat{x})>W_{\theta}(M)$ for all $M \in \mathcal{M}$. Firm $j$ can then gain by replacing all its menus with $\{\hat{x}(0,0)\}$.

To prove $(d)$, consider a strategy profile in the statement and suppose, by contradiction, that firm $i$ has a better response. Since each of the menus is offered by at least two firms, even if firm $i$ deviates, each type $\theta$ can choose $\left\{x_{\theta}^{*},(0,0)\right\}$ and guarantee an ex-ante utility of $H_{\theta}\left(x_{\theta}^{*}\right)$. Thus if firm $i$ can deviate profitably, there exist a menu $M$, a type $\theta$, and an offer $x \in \mathrm{BO}_{\theta}(M)$ such that $\Pi(x)>0$ and $W_{\theta}(M) \geq H_{\theta}\left(x_{\theta}^{*}\right)$. Since $x \in \mathrm{BO}_{\theta}(M)$, we obtain $W_{\theta}(M) \leq H_{\theta}(x)$ and hence $H_{\theta}(x) \geq H_{\theta}\left(x_{\theta}^{*}\right)$, which is a desired contradiction with $\Pi(x)>0$.

\section{Properties of equilibria}

\subsection{Ex-ante efficiency}

The equilibrium outcome is ex-ante efficient if there exists no menu $M$ that makes consumers of some type better off and generates higher profits for the firm: formally, there exists no menu-type-offer triple $(M, \theta, x)$ such that $x \in \mathrm{BO}_{\theta}(M), \Pi(x) \geq 0\left(=\Pi\left(x_{\theta}^{*}\right)\right)$, and $W_{\theta}(M) \geq H_{\theta}\left(x_{\theta}^{*}\right)$ with at least one inequality holding strictly. To see that the equilibrium outcome is ex-ante efficient, suppose, to the contrary, that there exists a triple $(M, \theta, x)$ that satisfies the above condition. Then

$$
H_{\theta}\left(x_{\theta}^{*}\right) \leq W_{\theta}(M) \leq H_{\theta}(x) .
$$

Therefore, $\Pi(x)=0$ and both inequalities in Eq. (13) hold with equality, which is a contradiction.

\subsection{Ex-post efficiency}

The equilibrium outcome is ex-post efficient if there exists no offer $x$ that makes consumers of some type $\theta$ better off in the ex-post stage (after the consumers have chosen their menus) and generates higher profits to the firm: formally, there exists no type-offer pair $(\theta, x)$ such that $\Pi(x) \geq 0$ and $U_{\theta}(x)+V_{\theta}(x) \geq U_{\theta}\left(x_{\theta}^{*}\right)+V_{\theta}\left(x_{\theta}^{*}\right)$ with at least one inequality holding strictly. If such an offer $x$ exists and the firm appends it to the menu chosen by the consumer, the consumer in the ex-post stage finds $x$ at least as good as any offer in the menu. ${ }^{3}$ Since $x_{\theta}^{*} \neq x_{\theta}^{U+V}$ is possible, the equilibrium outcome is generally not ex-post efficient. The firm does not want to include the ex-post Pareto improving offer in the menu since the offer is not desirable when evaluated with the consumer's commitment utility and if it is listed in the menu, the consumer will not choose the menu.

\subsection{Pooling}

The equilibrium outcome exhibits pooling if consumers of different types choose the same menu and the same offer: there exist a pair of distinct types $\left\{\theta, \theta^{\prime}\right\}$ and a menu $M \in \mathcal{M}$ such that

${ }^{3}$ This is based on a particular interpretation of the utility functions of Gul and Pesendorfer (2001): given a menu, the consumer prefers an offer that gives a higher value of $U+V$. 
$M \in \mathrm{BM}_{\theta}(\mathcal{M}) \cap \mathrm{BM}_{\theta^{\prime}}(\mathcal{M})$ and $x_{\theta}^{*}=x_{\theta^{\prime}}^{*}$. The following example shows that pooling is possible in a nontrivial sense. There are two types of consumers, A and B, and their utility functions are

$$
U_{\mathrm{A}}(q, t)=6 \sqrt{q}-t, \quad V_{\mathrm{A}}(q, t)=3(2 \sqrt{q}-t), \quad \text { and } \quad U_{\mathrm{B}}(q, t)=V_{\mathrm{B}}(q, t)=4 \sqrt{q}-t .
$$

There are two firms and the cost function is $C(q)=q$. Then $x_{\mathrm{A}}^{*}=x_{\mathrm{B}}^{*}=(4,4)$. A Nash equilibrium is where each firm offers a single menu $M=\{(4,4),(0,0)\}$.

\subsection{Upward temptation}

Let us say that consumers of type $\theta$ exhibit upward temptation if $q_{\theta}^{U}<q_{\theta}^{U}$. That is, temptation increases the marginal valuation for quality (or quantity). For these consumers, $x_{\theta}^{*}=x_{\theta}^{U}$, and hence the outcome is efficient for the $U$ utility. Note also that for these consumers, the equilibrium outcome is independent of their $V$ utility function. That is, upward temptation does not affect the consumers' equilibrium purchasing choice.

\subsection{Downward temptation}

Consumers of type $\theta$ exhibit downward temptation if $q_{\theta}^{V}<q_{\theta}^{U}$. That is, the marginal valuation is lower under temptation. For weight-loss programs, for instance, temptation lowers the marginal value of weight loss. For consumers with downward temptation, $x_{\theta}^{*}$ may differ from $x_{\theta}^{U}$, and hence the outcome is generally not efficient with respect to the commitment utility. This occurs because the consumers may be tempted to buy nothing: $V_{\theta}(0,0)>V_{\theta}\left(x_{\theta}^{U}\right)$ is possible. If the inequality holds and the consumers' expost choice is $x_{\theta}^{U}$, their ex-ante utility is at most

$$
U_{\theta}\left(x_{\theta}^{U}\right)+V_{\theta}\left(x_{\theta}^{U}\right)-\max \left\{0, V_{\theta}\left(x_{\theta}^{U}\right)\right\}=U_{\theta}\left(x_{\theta}^{U}\right)+V_{\theta}\left(x_{\theta}^{U}\right) .
$$

Since $x_{\theta}^{U}$ does not maximize $U_{\theta}+V_{\theta}$ on the $\Pi=0$ curve, there exists an offer $\hat{x}$ such that the menu $\{\hat{x}(0,0)\}$ gives the consumers a higher ex-ante utility and generates higher profits, in contradiction with equilibrium. Intuitively, the problem is the consumers' inability to commit to buy.

The same problem also causes some consumers to incur positive self-control costs in equilibrium even if all the equilibrium menus contain a single non-zero offer. Specifically, this occurs for consumers with downward temptation such that $V_{\theta}\left(x_{\theta}^{U+V}\right)<0$. Since $x_{\theta}^{*}=x_{\theta}^{U+V}$, the self-control cost is $V_{\theta}(0,0)-V_{\theta}\left(x_{\theta}^{U+V}\right)>0$.

For consumers with downward temptation, the equilibrium outcome depends on both the $U$ and $V$ functions. To see how the equilibrium outcome is affected by the $V$ function, suppose that the $V$ function can be decomposed as

$$
V_{\theta}(x)=\hat{V}_{\theta}(x) / w
$$

where $w>0$ is the index of willpower. Since $w$ is independent of $x$, it has no effect on the ordinal preferences of $V_{\theta}$, although it affects the ordinal preferences of $U_{\theta}+V_{\theta}$. For a larger $w$, the scale of $V_{\theta}$ is smaller and hence the influence of temptation on the actual choice is smaller. As $w \rightarrow \infty$, the willpower becomes infinitely strong in the sense that the maximizer of $U_{\theta}+V_{\theta}$ converges to the maximizer of $U_{\theta}$.

4 This definition is clearly a short cut. For a more satisfactory definition, see Esteban et al. (2003) or Esteban and Miyagawa (2004). 
The limit is the standard case with preferences given by $U_{\theta}$. It is easy to see that for any type, $q_{\theta}^{*}$ is nondecreasing in $w$. As the consumers' willpower gets stronger, the quality of goods sold gets higher.

\section{Participation fees}

The above analysis is based on the assumption that $(0,0) \in M$ for all menus. This assumption is violated if firms can charge "participation fees" (membership fees, entry fees, etc), so that even consumers who choose $q=0$ may have to pay a positive amount. If we remove the assumption $(0,0) \in M$, the equilibrium characterization is simplified considerably, as the following result shows. We omit the proof since it is virtually the same as that of Proposition $1 .^{5}$

Proposition 2. Suppose that firms can offer any compact subset of $\mathbb{R}_{+}^{2}$ as a menu. Then for any Nash equilibrium and any type $\theta$, (a) the ex-ante utility level of consumers of type $\theta$ is $U_{\theta}\left(x_{\theta}^{U}\right)$; (b) all consumers of type $\theta$ choose $x_{\theta}^{U}$; and (c) at least two firms offer menus $M$ such that $W_{\theta}(M)=U_{\theta}\left(x_{\theta}^{U}\right)$.Conversely, (d) a strategy profile is a Nash equilibrium if the set of menus is $\mathcal{M}=\left\{\left\{x_{\theta}^{U}\right\}: \theta \in \Theta\right\}$ and, for each type $\theta$, there exist at least two firms that offer $\left\{x_{\theta}^{U}\right\}$.

The equilibrium remains ex-post inefficient, but pooling does not arise (except in a trivial sense). The equilibrium purchasing pattern is completely independent of the $V$ utility functions, and the self-control cost is zero for all consumers.

\section{Acknowledgements}

We are grateful to an anonymous referee for helpful comments and Matthew Shum for his inputs.

\section{References}

Economides, N.S., 1985. A note on equilibrium in price-quality competition. Greek Economic Review 7, $179-186$.

Esteban, S., Miyagawa, E., 2004. Optimal menu of menus with self-control preferences, mimeo.

Esteban, S., Miyagawa, E., Shum, M., 2003. Nonlinear pricing with self-control preferences, mimeo.

Gul, F., Pesendorfer, W., 2001. Temptation and self control. Econometrica 69, 1403-1436.

Mandy, D.M., 1992. Nonuniform Bertrand competition. Econometrica 60, 1293-1330.

Maskin, E., Riley, J., 1984. Monopoly with incomplete information. RAND Journal of Economics 15, $171-196$.

Mussa, M., Rosen, S., 1978. Monopoly and product quality. Journal of Economic Theory 18, 301-317.

${ }^{5}$ Esteban and Miyagawa (2004) characterize optimal entry fees for the case of a monopoly, showing that they strictly improve profits for the firm. 